\title{
Gemeinschaft, Gesellschaft, Komplizenschaft. Alltagsethik im ländlichen China
}

Im Zuge der enormen politischen, gesellschaftlichen und kulturellen Veränderungen in der Volksrepublik China seit dem Ende des Maoismus haben viele Beobachter von einer moralischen Krise gesprochen. ${ }^{1}$ Die klassischen Prozesse der Moderne Urbanisierung und Industrialisierung, Individualisierung und Wertepluralismus scheinen in China in einer Geschwindigkeit und einem Ausmaß stattzufinden, der alles dagewesene übertrifft. Der gesellschaftlichen Wandels, so scheint es, lässt keine Zeit für eine Neu-ausrichtung geteilter Werte und Moralvorstellungen. In der chinesischen Öffentlichkeit werden häufig die Korruption der Regierung, der Niedergang traditioneller Werte, und die Konsumgesellschaft besprochen. Ereignisse wie der Fall des ehemaligen Parteisekretärs der Millionenstadt Chongqing, die gepanschte Säuglingsnahrung der Firma Sanlu, und v.a. die Indifferenz der Passanten im Fall eines Kleinkindes, das von mehreren Autos in Guangzhou überfahren wurde, stehen dabei stellvertretend für den allgemeinen moralischen Verfall.

In den Sozialwissenschaften versuchen sowohl chinesische als auch ausländische Forscher diesen moralischen Wandel auf verschiedene Weise zu analysieren.

Wenngleich es sich hier natürlich um allgemeine und vergleichbare

Modernisierungsprozesse handelt, spielen die besonderen Merkmale der chinesischen Geschichte und Gesellschaft eine zentrale Rolle - die relative Kontinuität der konfuzianischen Moral über Jahrhunderte, die Radikalität der Revolutionen des zwanzigsten Jahrhunderts und v.a. die Erfahrungen der maoistischen Ära. Ein Großteil der ethnologischen und soziologischen Forschung zum Wertewandel im China der Gegenwart betont dennoch die Veränderungen der letzten Jahrzehnte (z.B. Liu 2000, Yan 2003, Kleinmann et al 2011, Oxfeld 2011). In mancher Analyse ist dabei eine kulturpessimistische Einstellung, im Sinne eines Werteverfalls und moralischen Niederganges, kaum zu verkennen.

Hier möchte ich eine anthropologische und ethnographische Herangehensweise an die Ethik des Alltags in China vorschlagen, die auf die Verhandlung, Anpassung und Transformation von Ethik und Moral fokussiert. Ein solcher Ansatz steht jeder Klage über moralischen Niedergang diametral entgegen. Auf der Basis meiner LangzeitFeldforschung im ländlichen Hubei in Zentralchina beschreibe ich einen theoretischen Rahmen zur Analyse der Alltagsethik, der auf der Beziehung der Komplizenschaft basiert.

$$
* * *
$$

Wie viele andere Ethnologen habe ich einige frustrierende Erfahrungen während meiner Feldforschung gemacht. Was mich v.a. am Anfang meiner Zeit in der

\footnotetext{
${ }^{1}$ Dieser Artikel wurde zuerst im Ethnologischen Kolloqium der Universität Bayreuth vorgetragen. Ich bedanke mich bei Professor Kurt Beck für die Einladung und bei allen Teilnehmern für ihre Kommentare. Zentrale Argumente dieses Artikels wurden bereits in Steinmüller (2010) und (2013) in englischer Sprache veröffentlicht.
} 
Gemeinde Bashan in zentral China erstaunte, war die Antworten die ich oft auf bestimmte Fragen bekam. Zum Beispiel fengshui, die populäre Chinesische Kunst der Geomantik. Wörtlich übersetzt „Wind und Wasser", ist Fengshui eine Lehre der Harmonie zwischen astrologischen Zeichen und Merkmalen in der Landschaft, die ebenso mit den Geburtsstunden von Menschen in Beziehung gesetzt werden.

Fengshui-Experten werden oft zu Rate gezogen um die beste Lage für ein neues Haus oder das Grab eines Verwandten zu finden.

Als ich jedoch meine Nachbarn in Bashan in den ersten Tagen und Wochen nach meiner Ankunft fragte, ob sie denn an fengshui ,glauben würden“, oder einen fengshui-Experten bei einem Hausbau beispielsweise zu Rate ziehen würden, waren die Antworten meist verneinend. „Solche Sachen sind doch feudaler

Aberglaube.“ „Das mag es vielleicht in den ärmsten Bergregionen geben, aber hier in Bashan wirst Du das kaum finden.“

Nach einigen Wochen und Monaten in Bashan, wurde mir klar dass diese Antworten aber kaum etwas mit der Alltagspraxis dort zu tun hatten. Fast alle Familien die ein neues Haus planten oder eine Grabstelle für Verstorbene finden mussten, konsultieren einen fengshui Experten. Viele Menschen nehmen die Empfehlungen der fengshui Experten sehr Ernst, und folgen ihnen penibel. Nachdem ich selbst die Planung und den Bau einiger Häuser beobachtet hatte, mit vielen Menschen über die Bedeutung von feng shui für ihre Häuser diskutiert hatte, und von älteren Männern etwas mehr über die Details von fengshui und Divination gelernt hatte, änderten sich die Antworten auf dieselben Fragen. Da es ja den meisten Leuten im Dorf ganz klar war dass ich zumindest ein kleines bißchen über fengshui wusste, und - was grundlegend ist - verstand dass fast alle Familien in der Nachbarschaft fengshui-Experten beschäftigt hatten, würde es keinen Sinn mehr machen zu sagen dass fengshui einfach nur ,feudaler Aberglaube“ wäre der in Bashan kaum vorkommt.

Was die späteren Antworten dann charakterisierte war fast immer Indirektion oder Uneigentlichkeit. Im Sinne von ,ähh... Du weisst schon.“ „Soetwas wird oft als Aberglaube bezeichnet, aber..." oder einfach nur ein betretenes oder amüsiertes Lächeln.

Solche indirekten Reaktionen machen den Zuhörer der sie versteht zum Komplizen. Der Hintergrund dieser indirekten Reaktionen ist die Spannung zwischen offiziellem Diskurs und lokaler Praxis. Die charakteristische Uneigentlichkeit mit der sie geäußert werden reproduziert das geteilte Wissen und den Erfahrungshorizont einer lokalen Gemeinschaft.

\section{Komplizenschaft}

Die Unterschiede zwischen offiziellen und lokalen Diskursen, Öffentlichkeit und Privatsphäre, Zentrum und Peripherie, sind von entscheidender Bedeutung in jedem gesellschaftlichen Raum. Von der Soziolinguistik bis zur Politikwissenschaft bieten sich zahllose Theoriemodelle an um diese Gegensätze zu fassen. In der Soziologie sind beispielsweise die Schriften Erving Goffmans über Interaktion und „Gesichtsarbeit" (face-work) erwähnenswert (Goffman 1955). Für Goffmann war face-work eine der jeweiligen Zuhörerschaft angepasste Maske des Ich, die in 
spezifischen Situationen aufrecht erhalten will, da Individuen emotionell an Ihren Masken hängen und Ihre Berücksichtigung Kommunikation erleichtert. Was für die Selbstrepräsentation in der sozialen Interaktion relevant ist kann dann dann weiter auf allgemeine Differenzen zwischen der Privatsphäre und der Öffentlichkeit erweitert werden.

Jedoch sind Gegensätze zwischen dem öffentlichen Diskurs gegenüber Fremden und dem was in der Vertrautheit von Familien und Nachbarn gesagt werden kann vielleicht nicht immer so radikal wie in den Beispielen die ich eben angeführt habe. Die Unterschiede in diesen Beispielen haben etwas mit der Differenz zwischen der öffentlichen Sprache und lokalen Praktiken in einem bestimmten Kontext zu tun. Sie handeln von den jeweiligen Bewertungen von lokalen Praktiken in einem gesellschaftlichen Umfeld. Die Spannung zwischen lokaler Praxis und öffentlicher Bewertung ist in diesen Fällen radikal: was einerseits in der „Öffentlichkeit“ als rückständig verurteilt wird, ist andererseits unabdingbarer Bestandteil von lokaler Gemeinschaft.

Diesen Gegensatz beschreibt der Anthropologe Michael Heryfeld in seinem Begriff von „,kultureller Intimität“, den er folgendermaßen definiert: ,die Anerkennung der Aspekte einer kulturellen Identität die nach außen als peinlich empfunden werden, die Insider jedoch ihrer geteilten Sozialität versichern“ (2005:3). ${ }^{2}$ Die Spannung zwischen der Peinlichkeit nach außen, und der wichtigen Rolle die ebensolche Aspekte einer kulturellen Identität spielen können, produziert eine „kulturelle Intimität“, d.h., ein Sinn von Intimität der in einem kulturellen Spannungsfeld erzeugt wird.

Das scheint vielleicht etwas abstrakt, lassen Sie mich dies kurz an einem Beispiel von Michael Herzfeld illustrieren. In seiner Ethnographie The Poetics of Manhood (1985) diskutiert Herzfeld das Beispiel des Schafestehlens in den Bergen Kretas. Herzfeld hörte erst nach einigen Monaten in dem Bergdorf Glendi über diese Praktiken; die jedoch von ganz zentraler Bedeutung beispielsweise für lokale Allianzen und für die Performanz von Männlichkeit sind. Für Glendioten geht es in erster Linie nicht darum ob Schafe stehlen gut oder schlecht ist, sondern ob ein Mann gut oder schlecht im Schafe stehlen ist. D.h. ob die Art und Weise in der er vorgeht, sich gegenüber anderen und v.a. auch gegenüber der Polizei, verhält, und wie er seine Tat verbirgt oder darüber spricht. All das beschreibt Herzfeld als wichtigen Teil der Performanz von Männlichkeit in der lokalen Gemeinschaft. Was dem Schafestehlen zusätzliche Brisanz verleiht ist nicht nur das offizielle Verbot, sondern auch die Geringschätzung mit der Beamte und Städter solche Bräuche sehen. Das Schafe-stehlen zeichnet dabei genau den rückständigen und unzivilisierten Berghirten aus.

Das grundlegende Spannungsverhältnis auf dem die kulturelle Intimität beruht ist das von einem öffentlichen Diskurs und einer lokalen Praxis - der Unterschied von einer Gesellschaft von Fremden und einer face-to-face community. Im modernen Nationalstaat beruht diese Gesellschaft von Fremden auf Institutionen wie Schulen, Kasernen, Ämtern usw.; die kontinuierliche Interaktion mit Fremden in standardisierten Sprachen ermöglichen. Die Gesellschaft des Nationalstaates beruft sich ebenso auf eine gemeinsame nationalstaatliche Kultur, eine fixierte und

\footnotetext{
${ }^{2}$ Im Original: "the recognition of those aspects of a cultural identity that are considered a source of external embarrassment but that nevertheless provide insiders with their assurance of common sociality" (2005:3).
} 
gereinigte Essenz, die sich in der Moderne als grundsätzlich ebenbürtig und gleich mit anderen „Kulturen“ sehen muss. Was in lokale face-to-face communities getan und gesagt wird entspricht jedoch oft nicht der Kultur und Modernität des Nationalstaates, und manchmal steht es direkt im Widerspruch mit ihr. [zwei paragraph im langen, ab „Die Kunst des Schafestehlens“, nis „nationalstaatlichen Kultur“, einfuegen?]

Solche Spannungsverhältnisse werden in dem was Herzfeld ,soziale Poetik“ nennt beispielsweise die Kunst des Schafestehlens oder die Kunst Mann zu sein reproduziert. Ich versuche ähnliche Spannungen in China als Komplizenschaft zu beschreiben: charakterisiert durch ein (oft unausgeprochenes) Verständnis der Gegensätze dessen was lokal getan wird und was der weiteren Öffentlichkeit gegenüber repräsentiert wird. Solche Komplizenschaft tritt in Momenten zu Tage in denen gemeinschaftliche und gesellschaftliche Prinzipien aufeinanderprallen.

Kretische Hirten und Chinesische Bauern sind Mitwisser und Mittäter; nicht allein weil sie sich auf die Alltagsmoral einer lokalen Gemeinschaft beziehen, sondern weil diese Alltagsmoral mit der Sprache und den Werten ihrer nationalen Gesellschaft in Spannung und manchmal in Widerspruch steht. Die Praktiken der Komplizenschaft bezeichnen ein reflektives Engagement mit diesem Spannungsverhältnis - zu Komplizen werden nur die die mit beiden Seiten vertraut sind, mit der öffentlichen Repräsentation in der Gesellschaft und dem Erfahrungs- und Wissenshorizont der lokalen Gemeinschaft.

Im folgenden beschreibe ich solche Komplizenschaft im ländlichen China, in Bezug auf chinesische Geomantik (fengshui), Hochzeiten und Beerdigungen, und Lokalpolitik.

Die empirische Grundlage sind 18 Monate Feldforschung in dem Dorf Zhongba ${ }^{3}$ in den Wuling Bergen in der Provinz Hubei. Hubei ist mit neben Hunan, Anhui, Henan und Sichuan eine der Provinzen Zentralchinas, die noch weitgehend ländlich geprägt sind, und aus denen die meisten Arbeitsmigranten in die Großstädte und die Küstenregionen Südchinas kommen. Innerhalb Hubeis stellt die Bergregion des Südwestens, in der sich Zhongba befindet, eines der abgelegensten und wirtschaftlich unterentwickeltsten Gebiete dar.

\section{Fengshui}

Im Weiler der Yang Familie gibt es in 15 Häusern drei alte Männer die sich mehr oder weniger auf Astrologie und fengshui verstanden. Dies fand ich jedoch erst nach mehreren Monaten, und vielen Besuchen in den Häusern des Weilers heraus. Später würde Yang Minhui mir manchmal ausführlich das fengshui von bestimmten Häusern erklären. Diese Erklärungen waren oft einfache Beschreibungen von Bergen, Hügeln und Wasserläufen. Beispielsweise hat ein Haus idealerweise einen Berg hinter sich und einen sich schlängelnden Bach vor sich. Verständigungsprobleme beiseite, kamen diese Aussagen doch immer erst wenn ich mit der Familie ein gewisses Vertrauensverhältnis aufgebaut hatte. Und selbst dann riefen oft jüngere Familienmitglieder ihre Väter und Großväter ,zur Ordnung“; das heisst sie hielten die

\footnotetext{
${ }^{3}$ Alle Namen von Personen und Orten in der Provinz Hubei sind Pseudonyme.
} 
Alten an aufzuhören mit mir über fengshui zu reden oder machten sich direkt über sie lustig. Einige der alten Männer die etwas von fengshui verstanden wurden auch oft als „yin-yang“ ausgelacht.

Die Chinesische Geomantik - feng shui - ist das traditionelle Volkswissen von der Positionierung von Häusern und Gräbern in der Landschaft. Der fengshui Experte beschreibt eine Landschaft in seiner eigenen Spezialsprache als Kombination von Positionen und Strömen von Wasser und Energie, und findet mithilfe eines fengshui Kompasses die ideale Position für menschliche Behausungen (in der Großstadt auch von Wohnungen und Möbeln) in der Energieflüsse nicht geblockt werden und durch ihren Fluß zu Glück und Wohlstand führen. Der fengshui Kompass und die Symbolsprache die der Experte benutzt sind eng mit der volkstümlichen chinesischen Astrologie verbunden. ${ }^{4}$

In der chinesischen Geschichte wurden derlei Volksglauben oft mit Scharlatanen und Kurpfuschern in Verbindung gebracht. In den letzten zwei Dynastien des Kaiserreichs versuchte die Elite der Mandarine die Landbevölkerung vor allem durch Anleitung zu den rechten Bräuchen und Rituale zu regieren; dies beinhaltete manchmal auch das Verbot von Aberglauben und sektarischen Gruppen. Die kaiserliche Bekämpfung der Volksreligion war jedoch ungleich oberflächlicher als das was im 20. Jahrhundert in diesem Bereich geschah. Nicht zuletzt wurden fengshui und Astrologie ja auch von Mandarinen und vom Kaiser selbst für den Bau von Palästen und die Organisation von Palastritualen in Anspruch genommen.

Der Kampf gegen die Volksreligion wurde jedoch im 20. Jahrhundert ein wichtiger Teil der Modernisierungspolitik von Intellektuellen, Revolutionären und Parteikadern. Für die säkulare Moderne wurde der Volksglaube zum „Aberglaube“, eines der Zeichen für Chinas Schwäche gegenüber den westlichen Mächten. Die Eliten die für die „Stärkung Chinas“ und seine Modernisierung und Erneuerung arbeiteten wandten sich dabei nicht nur gegen den „Aberglauben“" sondern zunehmend auch gegen die Konfuzianische Tradition, insbesondere den Ahnenkult.

In Chinas langer Transition vom Kaisserreich zum Nationalstaat veränderte sich auch grundlegend die Position und Bedeutung der konfuzianischen Tradition. In den Worten des Sinologen Joseph Levenson war dies der Übergang von einer Zeit in der China die Welt war - einer Welt in der die konfuzianischen Klassiker die Grundlage jeder philosophischen und ethischen Diskussion waren - zu einer Zeit in der China in der Welt ist, und in der die Klassiker zu etwas wurden das die Partikularität der chinesischen Tradition auszeichnet, aber nicht mehr universelle Gültigkeit besitzt. ${ }^{5}$

\footnotetext{
${ }^{4}$ Stephan Feuchtwang (2001) beschreibt die Zusammenhänge von fengshui and Chinesischer Astrologie anhand einer detailierten Analyse der Symbole auf fengshui Kompassen und klassischen Almanachen: vom Gegensatz zwischen yin und yang über die fünf Elemente, über die acht Trigramme des Yijing, zu den zehn Himmelstämmen und den zwölf Erdweigen, ergibt sich ein transformatives System kosmischer Energieflüsse. Diese Elemente werden in fengshui und Divination mit den „Acht Zeichen“ der Geburtsstunde eines Menschen kombiniert (das sind zwei Zeichen je für Stunde, Tag, Monat und Jahr der Geburt). Ein Astrologe kann beispielsweise auf der Grundlage dieser Acht Zeichen die Tendenzen und Charaktereigenschaften eines Menschen bestimmen, was er an bestimmten Tagen tun und lassen sollte, welche Heirat unter einem guten oder schlechten Vorzeichen steht, und nicht zuletzt die Charakteristiken und Menschen und Landschaft in Harmonie bringen.

5 “.... in a genuinely Confucian China, a China that was the world, to cite the Classics was the very method of universal speech. The Confucian Classics were the repositories of value in the abstract,
} 
Während einige Intellektuelle in den letzten Jahren der Qing Dynastie die konfuzianische Tradition noch zu erneuern versuchten, gewannen später radikal antitraditionalistische Strömungen v.a. nach der Bewegung des 4.Mai (1919) die Oberhand. Die Neue Kulturbewegung propagierte westliche Ideale von Wissenschaft und Demokratie, eine auf der Alltagssprache basierende Standardsprache, und wandte sich explizit gegen die patriarchale konfuzianische Familienstruktur.

All diese neuen Ideen hatten in den 1920ern und 30ern noch wenig Einfluß auf das Alltagsleben der meisten Chinesen auf dem Land. Dieselben Ideen bereiteten jedoch den Grund für die kommunistische Revolution, die sie dann nach ihrem Sieg 1949 radikal in ganz China umgesetzt hat. In den Kampagnen unter Mao wurden Ahnenkult und Volksreligion dann als „,feudaler Aberglaube“ bekämpft. Hausaltäre und Ahnentafeln wurden verbrannt, in der Kulturrevolution wurden zahllose Tempel zerstört und Grabsteine zertrümmert.

Seit den 1980ern hat der Parteistaat die Kontrolle der Volksreligion zunehmend gelockert. In vielen Regionen wurden Tempel wieder aufgebaut, und Hausaltäre neu installiert. Jedoch bleiben sowohl Volksreligion, als auch volkstümlicher Konfuzianismus ambivalent. Viele ältere Menschen erinnern sich noch an die Auseinandersetzung in der Zeit des Maoismus, und damit bleibt vielen Elementen der Volksreligion und des volkstümlichen Konfuzianismus ein Hang von politischem Risiko. Die charakteristische Unsicherheit mit der sich viele deshalb auf diese Traditionen beziehen wird auch von Regierung und Partei geteilt. Es ist nicht immer klar was als „traditionelle Volkskultur“ akzeptabel ist und was als „feudaler Aberglaube" verurteilt werden muss.

Fengshui, beispielsweise, wird heutzutage in China auch manchmal in Architekturklassen unterrichtet. Die Rechtfertigungen die dafür bemüht werden, beziehen sich einerseits darauf dass dies „traditionelles“ chinesisches Wissen sei, und andererseits auch eine ,wissenschaftliche“ Grundlage habe. Zur selben Zeit ist es ganz klar dass diese Argumente nicht jeden überzeugen. An der Universität Wuhan, beispielsweise wurde 2009 ein Kurs zu fengshui angeboten; nach Protesten von besorgten Beamten und einer kurzen Diskussion in den lokalen Medien wurde der Kurs mit der Begründung abgebrochen dass es nicht Aufgabe der Universität sein könne Aberglauben zu verbreiten.

Nicht nur fengshui ist in dieser Weise äusserst ambivalent, sondern auch die zentralen Rituale des volkstümlichen Konfuzianismus, Hochzeiten und Beerdigungen.

\section{Hochzeiten und Beerdigungen}

Hochzeiten und Beerdigungen waren im vorkommunistischen China besondere Anlässe, in denen die zentralen Ideale des volkstümlichen Konfuzianismus verkörpert wurden: Respekt gegenüber den Ahnen, die hierarchischen Verhältnisse innerhalb der

absolute for everyone, not just Chinese values relevant to China alone. When the Classics make China particular instead of universal, it is a China in the world - still China, but really new, even as it invokes (indeed, precisely as it invokes) what connects it to the old." (Levenson 1965:xvii) 
Familie, und die Reziprozität von Gaben in Verwandtschaftsbeziehungen. Die konfuzianischen Prinzipien betonten vor allem Rollen und Beziehungen innerhalb der Familie, beispielsweise zusammengefasst in klassischen Prinzip der „3 Verbindungen und 5 Konstanten (das sind: der Mandarin dient dem Kaiser, der Sohn dient dem Vater und die Frau dient ihrem Ehemann. Die fünf Konstanten sind Güte, Gerechtigkeit, Ritual, Weisheit und Vertrauen). Menschen sollen den Rollen, Erwartungen, und angemessenen Handlungen der grundlegenden menschlichen Beziehungen, deren wichtigste die Beziehung zwischen Sohn und Vater ist, entsprechen. Dies ist prinzipiell immer der Fall, aber es muss zusätzlich in den rituellen Handlungen - volkstümlich v.a. eben Hochzeiten und Beerdigungen - betont werden.

Die wichtigsten rituellen Handlungen bei Familienfeiern finden immer in dem zentralen Raum der Bauernhäuser statt. Die Häuser in dieser Region haben einen Raum in der Mitte, dessen zentrale Achse (vom Hausaltar zum Haupteingang) nach den Prinzipien chinesischer Geomantik (Fengshui) ausgerichtet ist. Besonders bei Hochzeiten und Beerdigungen spielen sich die bedeutendsten Handlungen in diesem Raum, entlang der zentralen Linie des Hauses, ab: an Hochzeiten betreten Braut und Bräutigam gemeinsam diesen Raum und verbeugen sich gemeinsam vor dem Hausaltar. Bei Beerdigungen ist der Sarg genau entlang der zentralen Linie aufgestellt und der Raum selbst wird mit Bambusstangen, Papierrollen und Kränzen geschmückt und damit in eine „Halle der kindlichen Pietät““ (xiaotang) verwandelt.

Am Kopfende des Altars steht ein Tisch mit einem Foto des Verstorbenen. Hier bringen alle Verwandten ihre Geschenke und ehrweise dem Toten mit einem Kotau ihre Ehre. Bei Beerdigungen und anderen rituellen Anlässen wurde in der Vergangenheit von allen Mitgliedern der jüngeren Generation erwartet dass sie am Sarg des Verstorbenen einen neun-fachen Kotau vollführen. Diese traditionelle Form des Kotau bedeutet sich dreimal nieder zu knien, und sich dann jeweils drei mal (also insgesamt neun mal) zu beugen bis die Stirn den Boden berührt. Der Kotau drückt im volkstümlichen Konfuzianismus eben jenen so zentralen Respekt gegenüber den Ahnen aus. Er steht aber auch für die Untertänigkeit und Sklavenmentalität die Intellektuelle und Politiker am Konfuzianismus kritisierten.

Der amerikanische Anthropologe Andrew Kipnis hat den Kotau in Shandong in den 1990er als einen Ausdruck der „Bäuerlichkeit“ (peasantness) beschrieben (Kipnis 1995). Der Kotau der ländlichen Bevölkerung kann als Ausdruck ihrer Rückständigkeit oder des respektvollen und traditionellen Umgangs mit den Alten gesehen werden. In ethnographischen Beispielen beschreibt Kipnis genau diese Auseinandersetzungen im ländlichen Shandong.

Die Situation in Bashan in 2005 war ganz ähnlich. V.a. bei Beerdigungen wurde der Kotau aller anwesenden Verwandten aller jüngeren Generationen erwartet. Vielen Schülern und Studenten war dies aber etwas unangenehm, und sie führten den Kotau nur ganz flüchtig und nebenbei durch. Das heißt sie beugten sich nur drei Mal, anstatt sich auf den Boden zu werfen, und manchmal deuten sie auch die Verbeugung und die gefalteten Hände dabei nur an, anstelle sie ganz auszuführen.

Wie der Kotau werden andere Elemente von Beerdigungen als unangenehm und peinlich empfunden, v.a. In der Präsenz von Städtern und Fremden wie mir. Die 
ausführlichen Rituale und insbesondere der Kotau stehen hier klar für Ländlichkeit und Tradition und potentiell auch Rückständigkeit.

Während solche Details ritueller Symbolik wahrscheinlich eher den Menschen bewusst sind die längere Zeit in den Städten verbracht haben, werden andere Aspekte tagtäglich diskutiert. Das sind primär das Essen und die Geldgeschenke an solchen Anlässen. Diese, und die Anzahl von Gästen und von freiwilligen Helfern (meist Nachbarn und nahe Verwandte) spiegeln dabei klar den sozialen Status der gastgebenden Familie wieder.

Während vor der kommunistischen Revolution viele dieser Rituale, und v.a. grosse Geldgeschenke und Banquette nur wenigen reichen Familien vorbehalten war, sind sie heutzutage unabdinglicher Bestandteil von Hochzeiten und Beerdigungen in allen Familien. Ermöglicht wurde dies durch das Wachstum von Lohnarbeit und Warenökonomie seit den 80ern. Anfang der 80er konnten sich nur wenige Familien große Familienfeiern leisten und Geschenke bestehen meist aus Naturalien. Diese wurde seither zumindest durch Geld ersetzt und die Summen die gegeben werden haben sich in den letzten zwei Jahrzehnten drastisch vervielfacht. In den 80er Jahren kamen noch Geschenke von einigen Fen vor (zehn Fen sind ein Yuan Renminbi). Zum Ende der 1990er waren es einige Yuan, und während meiner Feldforschung zwischen 2005 und 2007 war die Minimalsumme bei 20 Renminbi.

Diese Inflation bedeutet für viele Familien eine schwere finanzielle Last. Falls eine Familie zu einer Feier eingeladen wird, ist es so gut wie unmöglich abzulehnen. Nicht zu kommen, oder wenigstens ein Geschenk zu senden, wird als Beleidigung angesehen und kann leicht das Ende einer Beziehung bedeuten. Für viele Familien beliefen sich die jährlichen Ausgaben für solche Geldgeschenke in den Jahren 2005 und 06 auf einige tausend Renminbi, und damit manchmal bis auf ein drittel ihrer gesamten jährlichen Ausgaben - zumindest war das oft eine Schätzung die mir gegenüber gegeben wurde. Die meisten Familien führen genau Buch über diese Ausgaben, und nach den wenigen Bilanzen die mir in Nachbarsfamilien gezeigt wurden, und die mir ihre anderen jährlichen Ausgaben vorrechneten, scheint mir dieser Anteil realistisch.

Wenn man oder frau nun jedes Jahr so viel Geld ausgibt, verstärkt das natürlich die Hoffnung selbst bald in einer Feier dieses Geld wieder zurück zu erhalten. Mit Blick auf die Bilanzen, über die in den meiste Familien die Frauen Buch halten, werden dann all diejenigen eingeladen die selbst in den letzten Jahren beschenkt wurden. Es kann davon ausgegangen werden dass die Geldgeschenke die zurückgegeben werden nie niedriger als das letzte Geschenk sein können.

Weil es jedoch in jeder Familie nur eben eine begrenzte Anzahl Hochzeiten und Beerdigungen geben kann, haben mehr Familien auch andere Anlässe genommen um Gäste einzuladen. Die Einweihung eines neuen Hauses war dafür besonders geeignet, wurden doch genau in den letzten Jahren zahllose neue Häuser gebaut. Daneben werden auch die Geburtstage von älteren Familienmitgliedern, bestandene Aufnahmeprüfungen für die Universität, die Aufnahme von Söhnen ins Militär und andere Anlässe gefeiert. 
Angeblich gab es auch Leute die beispielsweise die Erreichtung eines neuen Schweinestalls feierten - was lokal als ein „Wein für ein Nicht-Ereignis“ (wu shi jiu) genannt wird. Derlei Weine werden auch gern kritisiert; und doch ist es für Verwandte, Nachbarn und Freunde auch hier kaum möglich Einladungen abzulehnen.

Seit einigen Jahren gab es immer wieder Zeitungsberichte und kleinere Parteikampagnen gegen die ,verschwenderische und feudale“ Sitte für alle möglichen Anlässe Feiern abzuhalten, um viele Geldgeschenke einzukassieren. Die Kampagnen werden aber oft nur halbherzig durchgeführt und hatten kaum Einfluss auf die lokalen Praktiken. Grundlegend war hierfür dass neben Geschäftsleuten vor allem mittlere Parteibeamte große Familienfeiern abhalten. Mehr als andere sind lokale Parteibeamte auf gute persönliche Beziehungen angewiesen, und sehen sich wie viele andere dem Zwang gegenüber jedes Jahr auf zahlreichen Feiern zu erscheinen. Im Sommer 2011 beschloss die Regierung der Präfektur Enshi deshalb diese ,verschwenderischen Feiern“ radikal zu unterdrücken. In einigen Dörfern Bashans veranstalteten Beamte der Bezirksregierung Razzien auf Geburtstagsfeiern und Feiern für bestandene Aufnahmeprüfungen (also „Nicht-Ereignisse“) und beschlagnahmten die bereits eingenommenen Geldgeschenke.

Während meiner Zeit in Bashan wurde ich oft auf Hochzeiten und Beerdigungen eingeladen. Anfangs hatten Beamten die für mich zuständig waren mich auch einige Male ermutigt auf diese Feiern zu gehen, wo ich „lokale Kultur“ sehen könnte.

Jedoch war es einigen höhergestellten Beamten auch sichtbar unangenehm, dass ich auf so vielen Feiern zu sehen war, insbesondere auf denen von Parteimitgliedern und Beamten. Am Anfang meiner Feldforschung akzeptierte ich bereitwillig so gut wie alle Einladungen zu Hochzeiten und Beerdigungen, auch wenn sie nicht direkt vom Gastgeber kamen. Auf diese Weise nahm ich an einigen Feiern teil bei denen ich die gastgebende Familie gar nicht kannte.

Eine von diesen Familien war die Familie von Sekretär Sun, eines hochrangigen Beamten in der Landkreisregierung, der bis vor einigen Jahren der Parteisekretär von Bashan war. Als ehemaliger Parteisekretär war er eine zentrale Figur in Bashan gewesen und jetzt die perfekte Verbindung von Bashan in den Landkreis.

Die Beerdigung seines Vaters war die größte Beerdigung die ich in Bashan in ein einhalb Jahren gesehen hatte. Meine Nachbarn waren als Musiktruppe mit Suona und Trommeln eingeladen und hatten mir davon erzählt. Ich könne doch mitkommen und dem alten Parteisekretär von Bashan meine Ehre erweisen.

Das Bauernhaus der Familie lag in einem Dorf das sehr weit vom Marktplatz von Bashan in den Bergen lag. Auf dem Weg, säumten sich bereits schwarze Audis und Volkswagen - ich zählte an die 30. Ein großer Teil der lokalen Parteiprominenz und viele Geschäftsleute gaben sich die Klinke in die Hand.

Die Feierlichkeiten dauerten die ganze Nacht, es kamen mehrere Gruppen von Ritual experten, Tänzern und couplet Sängern. Wärhend der des Abends zählte ich ungefähr 30 ,luogu dui“, Suona-Musiktruppen (gesendet von den „Töchtern“ der Familie, das heisst allen Töchtern und weiblichen Cousinen der Patriline, mit ihren Ehemännern). An solchen Feiern werden Geldgeschenke immer in roten Büchern vermerkt - hier 
reichten drei volle Bücher nicht aus. Die volle Summe waren ca. 70000 Yuan (mehr als 9000 Euro) - zu einer Zeit in der das Jahreseinkommen der Nachbarnn nach Landkreisstaistik bei ca. 4000 Yuan lag.

Während des Abends sprach ich mit Nachbarn und einigen der Verwandten des Parteisekretärs, die sehr interessiert waren über Deutschland und England zu hören.

Der Parteisekretär selbst jedoch wandte kein Wort an mich - obwohl ich auf Auffoderung eines Organisators sogar eine kleine Rede hielt, in der ich mein Interesse an der lokalen Kultur ausdrücken sollte. Er selbst hattte den ganzen Abend über einen grünen Militärmantel an, genau wie sein Bruder, der den Bauernhof der Eltern bewirtschaftete. Die Grabrede, die der Parteisekretär am Abend hielt, handelte vom harten Leben auf dem Land, was sein Vater alles durchgemachte hatte, und dass er sein ganzes Leben lang ein treues Parteimitglied gewesen sei.

Die Beerdigung war Hauptthema der Gespräche am Marktplatz und in den Häusern Bashans für die nächsten paar Tage. Einige Beamte sprachen mich darauf an, ob ich nicht meinte dass sich so eine große und verschwenderische Feier nicht für einen Parteibeamten zieme?

Die besten Gespräche jedoch hatte ich mit Nachbarn und Freunden. Der pensionierte Lehrer Song erzählte mir dass dies die größte Beerdigung gewesen wäre die je in Bashan stattgefunden hätte. Man sagte sich dass der Parteisekretär jetzt etwas besorgt war, daß er bei höheren Stellen angezeigt werden könne. Noch dazu war ich, der ausländische Gast des Landkreises, bei der Beerdigung aufgetaucht. Jedem waren Fälle bekannt in denen lokale Beamte wegen solchen Feiern angeklagt wurden, Amt und Parteimitgliedschaft verloren hatten und sogar im Gefängnis gelandet waren.

Song lies seinem Unmut freien Lauf: die $70000 \mathrm{RMB}$, die ich in den roten Büchern gesehen hatte, wären noch lange nicht alles. So gut wie alle höheren Beamten in Bashan müssten etwas an Sun geben; nur um sich ihrer guten Beziehung zu diesem mächtigen Beamten zu versichern. Andererseits war so ein Geschenk zur Beerdigung des Vaters auch ein willkommener Vorwand um dem Parteisekretär Geldgeschenke zukommen zu lassen. Viele würden es vorziehen dass privat zu tun, statt in den Büchern aufzutauchen, und von daher geht Song davon aus dass die wirkliche Summe ein vielfaches von den 70000 RMB in den roten Büchern war.

Sowohl die Lokalbevölkerung als auch Regierungsbeamte sind sich der Bedeutung der Familienfeiern und der Schwierigkeiten diese zu verbieten, bewußt. Zur selben Zeit wissen sie auch dass viele Elemente dieser Feiern im öffentlichen Diskurs negativ beschrieben werden, und zwar als Aberglaube, Verschwendung und Rückständigkeit.

Die Art und Weise wie sich der Parteisekretär am Abend verhielt - inklusive seiner Kleidung, die seine bäuerliche Herkunft betonte; die Reaktionen der Kollegen in der lokalen Regierung, von denen die meisten Geldgeschenke gegeben hatten, und die 
doch insgeheim über die Geldmengen spekulierten und manchmal auch das Risiko einer solchen Feier betonten; und nicht zuletzt die privaten Äußerungen von Lehrer Song, der deutliche Worte für den dekadenten Charakter einer solchen Feier fand - als das zeugt von einer Komplizenschaft innerhalb einer lokalen Gemeinschaft.

Diese charakteristische Indirektheit spielt auch in der Politik - im engeren Sinne des Wortes Politik - eine zentrale Rolle.

\section{Lokalpolitik und Korruption}

Ein großes Thema vieler Konversationen, die ich in Zhongba hatte war die Korruption der lokalen Beamten. Typische Geschichten handelten vom Aufstieg und Fall von Parteibeamten; sowohl in den lokalen Behörden von Bezirk und Präfektur als auch in den höhreren Ebenen von Provinz und Nation - letztes Jahr wurde auch der Fall des Parteichefs von Chongqing, Bo Xilai, ausführlich kommentiert. Aber während diese Ebenen weit von der Alltagsrealität der Bauern in Bashan entfernt sind haben die lokalen Beamten, v.a. in Dorf, Gemeinde, und Landkreis, direkt Einfluß auf Angelegenheiten die von unmittelbarem Interesse für die Lokalbevölkerung sind. In der Art und Weise in der sich die Bauern von Zhongba auf die verschiedenen Ebenen der Partei- und Regierungshierarchie beziehen spielt die Komplizenschaft von und mit Offiziellen eine entscheidende Rolle.

Die lokale Regierung im ländlichen China wurde in den letzten Jahrzehnten grundlegend reformiert. Administrative Einheiten wurden mehrmals umorganisiert und zusammengelegt, Einstellungsverfahren und Kontrolle von Parteikadern wurden „modernisiert" und sollten transparenter gemacht werden; in den Dörfern werden seit 1998 ,demokratische“ Wahlen für Dorfvorsteher und Gemeinderäte abgehalten. Es ist jedoch noch nicht absehbar bis zu welchem Grad all diese Reformen wirklich implementiert wurden. Sowohl die institutionelle Trägheit der Partei- und Staatsorgane, als auch lokale Allianzen und Einfluss bremsen die Reformen und machen Reformprogramme oft zu blossen Lippenbekenntnissen.

In Zhongba beisielsweise ist jedem Bauern klar, dass die „demokratischen“ Dorfwahlen nicht ganz demokratisch sind. Obwohl hier schon einige Male Dorfwahlen abgehalten wurden, werden für die wichtigen Positionen des Gemeindevorstehers und des Parteisekretärs immer Personen ausgewählt, die in der Gunst der nächsthöheren Partei- und Regierungsebene im Landkreis stehen. Solche Amtsträger werden in Wirklichkeit ,von oben geschickt", auch wenn vorgegeben wird, dass sie in freier und geheimer Wahl gewählt wurden.

Ähnlich verhält es sich mit dem Programm des „Aufbaus einer neuen sozialistischen Landwirtschaft" das in Zhongba in den letzten Jahren durchgeführt wurde. Innerhalb dieses Programmes werden mit staatlichen Subventionen Entwicklungsprojekte auf lokaler Ebene gefördert. Dies steht im grösseren Rahmen der nationalen Agrarpolitik, in der unter dem selben Namen (der ,neuen sozialistischen Landwirtschaft") in den letzten Jahren die Steuerlast für die Bauern deutlich gesenkt, die pauschale Grundsteuer abgeschafft und stattdessen eine Subventionierung der Landwirtschaft begonnen wurde. 
Das Projekt des „Aufbaus einer neuen sozialistischen Landwirtschaft“ in Zhongba beinhaltet u.a. workshops für Tee-Anbau und Marketing, Fortbildungskurse für den Umgang mit Computern und den Ausbau der Dorfstrasse. Der größte Teil der Gelder wurde jedoch auf die Renovation und Verschönerung von Bauernhäusern verwendet. So bekamen ausgewählte Haushalte z.B. Beihilfen um die Fassaden ihrer Häuser zu streichen, Schriftrollen an den Hausrahmen anzubringen und die Vorhöfe zu betonieren. Die Auswahl der geförderten Haushalte wird nach Kriterien getroffen, die den meisten Dorfbewohnern bekannt sind: Zum einen die Entfernung, ja, die Sichtbarkeit, von der Asphaltstrasse die durch das Dorf führt, und zum anderen die Nähe bestimmter Haushalte zu Beamten in der Dorf- und Landkreisregierung.

Viele Menschen im Dorf bezeichnen deshalb diese Subventionsprogramme als „Gesichtsprojekte“ (mianzi gongcheng), also Projekte, die nur darauf ausgelegt sind das „Gesicht“ der Lokalregierung zu wahren. Diese muss nämlich ihre Arbeit in der „ländlichen Entwicklung“ regelmässig Beamten von höheren Regierungsebenen präsentieren, die zu Inspektionsvisiten in das Dorf kommen.

Klagen über die Ungerechtigkeiten in der Verteilung der Subventionen waren dabei an der Tagesordnung. V.a. Bauern die ihre Häuser fernab von der Teerstrasse Zhongbas hatten und keinerlei Hoffnung auf staatliche Förderung hatten, gaben ihrem Unmut oft Ausdruck. „Die Regierung hier dient den Reichen und nicht dem Volk“ eine Aussage die den Spruch Maos ,dem Volk dienen“ (wei renmin fuwu) auf den Kopf stellt.

Diese Kritik wird jedoch nicht von allen geteilt und ist abhängig von der Situation. Wenn höhere Beamte anwesend sind wird sie kaum geäußert und genauso hängt sie von den Interessen der jeweiligen Beteiligten ab; diejenigen Haushalte, die Beihilfen erhielten, kritisierten die Lokalregierung natürlich weniger.

Zur selben Zeit kann der Diskurs über „Gesichtsprojekte“ auch den lokalen Beamten dazu dienen, ihre eigenen Unzulänglichkeiten zu benennen. Der Parteisekretär von Zhongba, beispielsweise, Xiang Tao, war jemand der direkt in der Implementierung der lokalen Entwicklungsprojekte arbeitete. Erst Mitte zwanzig, wurde ihm bereits die Stelle des Parteisekretärs in diesem Dorf anvertraut - eine priviligierte Position, die glänzende Aufstiegschancen verprach, die allerdings auch mit einigen Herausforderungen verbunden war.

Nach einem Jahr in Bashan war ich mit Xiang Taos Klagen über die Dilemmata seiner Position vertraut geworden. Einerseits kamen Dorfbewohner zu ihm die Subventionen für ihre Haushalte einforderten und sich über die Ungerechtigkeiten der Verteilung beklagten. Anderseits musste er die Beamten an höherer Stelle zufriedenstellen. Die Organisation von Besuchen und Ortsaufnahmen höherer Regierungsstellen im „Modelldorf“" nahm dabei einen großen Teil seiner Arbeitszeit in Anspruch. Und wenn Kollegen ihn auch manchmal zu seiner Arbeitsstelle im „Modelldorf“ beglückwünschten und ihn um seine Aufstiegschancen beneideten, konnte er doch auch oft nur die „Gesichtsprojekte“ verfluchen, die er durchführen musste. Oder einfach zugeben dass die „Konstruktion einer sozialistischen neuen Landwirtschaft" hier eben grundsätzlich ein „Gesichtsprojekt" war. Das heißt, dass die Subventionen nur dazu verwendet werden, ausgewählte „Modellhaushalte“ zu fördern, und dass mehr kaum möglich wäre, unter dem ständigen Druck lokale 
Erfolgsprojekte vorweisen zu müssen. Derlei Eingeständnisse seinerseits habe ich jedoch nur in privaten Kontexten, d.h. in der Präsenz von Kollegen und Freunden, gehört.

Diese „Gesichtsprojekte“ sind in einer Hinsicht Potemkinsche Dörfer, die dazu errichtet werden um eine Fassade der ländlichen Entwicklung für höhere Regierungsebenen zu schaffen. Andererseits zeigen die verschiedenen Arten und Weisen in denen Bauern und Beamte die Subventionen behandeln und das Für und Wider der Entwicklungsprogramme aushandeln, wie die Implementierung solcher nationaler Politik auf lokaler Ebene funktioniert. - und zwar ganz grundlegend in einer Politik von Komplizen, die die Mechanismen der Gesichtswahrung verstehen.

\section{Zusammenfassung}

Ich habe diesen Artikel mit der Idee einer Komplizenschaft begonnen, die auf dem geteilten Wissen und dem Erfahrungshorizont einer lokalen Gemeinschaft beruht. In meiner eigenen Erfahrung war das besonders klar bei Dingen wie fengshui - wo sich die Reaktionen auf meine Fragen am Anfang und später in der Feldforschung ganz klar unterschieden.

Ähnliches trifft auf Familienfeiern und Lokalpolitik zu. Erfahrungen wie diese sind gewiss typisch für ethnographische Feldforschung. Sie sind jedoch genauso relevant für die Bewohner Zhongbas, die sich je nach Situation für bestimmte Haltungen gegenüber fengshui, ritual, und Austausch entscheiden müssen. In all diesen Bereichen finden wir Spannungen und Widersprüche zwischen offiziellem Diskurs und lokaler Praxis. Diese sind jedoch nicht statisch - sondern beidererseits wandelbar und in gewissem Grade flexibel.

Die Botschaften von Staat und Partei sind heutzutage nur mehr selten uneindeutig und die Spannungen und Widersprüche, die sich z.B. aus der maoistischen und konfuzianistischen Perspektive auf Beerdigungsrituale oder dem öffentlichen Diskurs über Glücksspiel und dessen lokalen Praxis ergeben, weisen auf einen Raum kultureller Intimität hin: Eine lokale Gemeinschaft des impliziten Verständnisses. Mein Begriff der „communities of complicity“ ist weder eine Romantisierung lokaler Gemeinschaften die Partei und Staat Widerstand leisten, noch eine Idealisierung der Macht letzterer. Stattdessen beschreibe ich wie Menschen in alltäglichen Handlungen öffentliche Diskurse und lokale Praxis kreativ zusammenbringen, und sich dabei auf eine lokale Gemeinschaft beziehen.

Zum Schluss ein paar Vergleiche mit anderen Beschreibungen der Spannungen zwischen lokaler Gemeinschaft und dem Staat in China. Beispielsweise wurde der lokale Staat in China von manchen Politikwissenschaftlern und Anthropologen als „neotraditionalistisch“ beschrieben (Walder 1986). Das heisst, eine Bürokratie die sich außen eine moderne Fassade von Transparenz und Rationalität gibt, während innerhalb traditionelle Prinzipien von persönlichen Beziehungen und Patronage herrschen. Historische Prozesse der „state formation“ wurden in diesem Stil als „Involution“" gefasst, dass heisst als Prozesse die zu einer Verstärkung der patronage Beziehungen innerhalb der Staatsmaschine führten (Duara 1987; Bray 1994). 
Die Analysen des „Neo-traditionalismus“ im Chinesischen Staat, die einige Politikwissenschaftlern vorgeschlagen haben zeigen klar wie sich „traditionelle“ Strukturen und Bindungen während des 20. Jahrhunderts transformiert haben, und beispielsweise in veränderter Form lokale Regierung auf dem Land (Shue 1990) oder Arbeitseinheiten in Fabriken weiter existeren (Walder 1986). Diese Entwürfe ignorieren jeodoch die Art und Weise wie sich lokale Akteure sowohl auf modernistische öffentliche Diskurse als auch auf die Modelle einer lokalen Gemeinschaft beziehen. Während in den Involutionsmodellen die traditionalistischen Patronage die Realität ist, und der modernistische Diskurs nur Fassade, versuche ich durch die Beschreibung der Komplizenschaft die Relevanz beider im Alltagsleben zu beschreiben. Das heisst anzuerkennen das sowohl modernistische als auch traditionalistische Diskurs-Praktiken soziale Wirklichkeit sind und von Akteuren alltäglich verbunden, kontrastiert und ausgehandelt werden.

Auf der anderen Seite gibt es bei Anthropologen und vor allem chinesischen Ethnologen auch Tendenzen zur Romantisierung lokaler Gemeinschaften, beispielsweise wenn die Revitalisierung von lokalem Ritual als Beweis für die Beständigkeit von lokaler Gemeinschaft und Tradition interpretiert wird. Demgegenüber betont der Fokus auf die Komplizenschaft die Geschichtlichkeit von Ritual und „Tradition“.

In diesem Sinn stellt die Produktion der Komplizenschaft auch möglicherweise einen allgemeinen theoretischen Beitrag zur ethnographischen Methode dar. Obwohl meine Beispiele meist aus China kamen, und vor allem vom Gegensatz eines modernistischen öffentlichen Diskurses und traditionialistischer lokaler Praxis handelten, ist die Komplizenschaften die ich beschrieben habe in keiner Weise einzigartig für China. Im Gegenteil, sie lässt sich auch als Mittler im klassischen Gegensatzpaar von „Gemeinschaft" und „Gesellschaft“" verstehen. In diesem Sinne lassen sich interessante Parallelen für mein Argument in einem klassischen Aufsatz zu diesem Gegensatzpaar finden. In seiner Schrift von den „Grenzen der Gemeinschaft“ (2002, Erstveröffentlichung 1924) stellt Helmuth Plessner die Risiken der „Gemeinschaft“ dar. Für ihn ist eine vollkommene Einbindung in eine Gemeinschaft unmöglich, da dies der „Doppelnatur des Menschen“ widerspricht. Innerlichkeit - äusserlichkeit; da der Mensch nie ganz über seine eigene Innerlichkeit bescheid weiss, muß manches unausgesprochen blieben.

Sein Gegenvorschlag ist ein „Gesellschaftethos“, das heisst ein Ethos des „SichFügens" in die Erfordernisse einer Gesellschaft, in der Fremde aufeinandertreffen die niemals alle Werte und Erfahrungshorizonte teilen. Plessner schreibt man müsse die Hoffnung aufgeben „daß einmal überall mit offenen Karten gespielt werden“ kann (2002:12). Stattdessen betont er betont die Rolle von „Distanz“, „Spielen“, „Zeremonie“, und v.a. „Diplomatie“ und „Takt". All diese Former der Indirektion und Un-eigentlichkeit ermöglichen die Interaktion von einander Fremden in einer Gesellschaft. Plessners Aufsatz hatte dabei auch klar den aufstrebenden Sozialismus im Auge, und sah deutlicher als manch andere die Gefahren eines aufotroyierten Gemeinschaftsideals.

Mein Ansatz hier kommt von der anderen Seite des Gegensatzes von Gemeinschaft und Gesellschaft. Als Ethnologe und Feldforscher ist mein Ausgangspunkt eine lokale 
Gemeinschaft in der sich die meisten Leute kennen. Von dieser Gemeinschaft ausgehend habe ich versucht die „Grenzen der Gesellschaft“ im ländlichen China aufzuzeigen, d.h. die Grenzen dessen was im öffentlichen Diskurs zwischen Fremden mittelbar ist. Im Zwischenraum von Gemeinschaft und Gesellschaft, oder besser, von Vergemeinschaftung und Vergesellschaftung, stoßen wir dann - wie Plessner - auf Indirektionen. Der Fokus in meinem Argument lag dabei auf der Komplizenschaft; der Mitwisserschaft lokaler Akteure die sich ihres gemeinsamen Standes zur fremden Gesellschaft bewußt sind. Die Komplizenschaft ist dabei Plessners Schlagworten Distanz, Zeremonie, und Takt nicht grundsätzlich unähnlich. Sowohl die Reaktionen der Komplizenschaft als auch des Taktes betonen die Uneigentlichkeit der Kommunikation. Ihre Stoßrichtung ist jedoch eine andere: Während Höflichkeit und Takt die gesellschaftlich Interaktion ermöglichen, erhalten die Reaktionen der Komplizenschaft die gemeinschaftliche Interaktion aufrecht.

Die Indirektion und Uneigentlichkeit dieser Arten von Kommunikation beinhaltet ein reflektives Element: Akteure müssen sich unterschiedlicher Sprachregister und Wertevorstellungen bewusst sein - beispielsweise der Gegensätze von offzieller Parteirhetorik und den Erwartungen von Nachbarn und Verwandten bei Familienfeiern.

Wenn wir moralisches Handeln als Bezug auf Werte verstehen, und Ethik als Reflektion der Moral - dann lassen sich die uneigentlichen Handlungen der Komplizenschaft als ethisch bezeichnen. Das heisst nicht als eine systematische Reflektion verschiedener Moralsysteme, sondern als implizite Reflektion im Alltag. Zwischen Gemeinschaft und Gesellschaft, steht damit die Komplizenschaft für eine Ethik des Alltags - nicht ein von Philosophen erfordertes Ethos einer modernen Gesellschaft, sondern eine Ethik die ethnographisch gefunden wird. 


\section{Bibliographie}

Bray, Francesca

1994 The Rice Economies: Technology and Development in Asian Societies.

Berkeley: University of California Press.

Duara, Prasenjit

1987 State Involution: A Study of Local Finances in North China, 1911-1935.

Comparative Studies in Society and History 29 (1): 132-61.

Feuchtwang, Stephan

2001 An Anthropological Analysis of Chinese Geomancy. Bangkok: White Lotus.

Goffman, Erving

1955 On Face-work: An Analysis of Ritual Elements of Social Interaction.

Psychiatry: Journal for the Study of Interpersonal Processes 18 (3): 213-231.

Herzfeld, Michael

1985 The Poetics of Manhood: Contest and Identity in a Cretan Mountain Village.

Princeton: Princeton University Press.

Herzfeld, Michael

1993 The Social Production of Indifference: Exploring the Symbolic Roots of

Western Bureaucracy. Chicago: University of Chicago Press.

Herzfeld, Michael

2005 Cultural Intimacy: Social Poetics in the Nation-state. New York: Routledge.

Kipnis, Andrew B.

1995 Within and Against Peasantness: Backwardness and Filiality in Rural China.

Comparative Studies in Society and History 37 (1): 110-135.

Kleinmann, Arthur, Yunxiang Yan, Jun Jing, Sing Lee, Everett Zhang, Tianshu Pan, Fei Wu, and Jinhua Guo

2011 Deep China. The Moral Life of the Person. What Anthropology and Psychiatry Tell Us About China Today. Berkeley: University of California Press.

Levenson, Joseph Richmond

1965 Confucian China and Its Modern Fate: A Trilogy. Berkeley: University of California Press.

Liu, Xin

2000 In One's Own Shadow: An Ethnographic Account of the Condition of Post-

Reform Rural China. Berkeley: University of California Press.

Oxfeld, Ellen

2010. Drink Water, but Remember the Source. Moral Discourse in a Chinese Village. Berkeley: University of California Press.

Plessner, Helmuth 
2002 Grenzen der Gemeinschaft: Eine Kritik des Sozialen Radikalismus. Frankfurt a.M.: Suhrkamp.

Shue, Vivienne

1990 The Reach of the State: Sketches of the Chinese Body Politic. Stanford:

Stanford University Press.

Steinmüller, Hans

2010 Communities of Complicity: Notes on State Formation and Local Sociality in Rural China. American Ethnologist 37 (3): 539-49.

Steinmüller, Hans

2013 Communities of Complicity: Everyday Ethics in Rural China. New York:

Berghahn.

Walder, Andrew G.

1986 Communist Neo-Traditionalism Work and Authority in Chinese Industry.

Berkeley: University of California Press.

Yan, Yunxiang

2003 Private Life Under Socialism: Love, Intimacy, and Family Change in a Chinese Village, 1949-1999. Stanford: Stanford University Press. 